\title{
Population genetics of Plasmodium falciparum and Plasmodium vivax and asymptomatic malaria in Temotu Province, Solomon Islands
}

\author{
Karen-Ann Gray ${ }^{1,2}$, Simone Dowd ${ }^{1,2}$, Lisa Bain ${ }^{1,2,3}$, Albino Bobogare ${ }^{4}$, Lyndes Wini ${ }^{4}$, G Dennis Shanks ${ }^{1,2}$ \\ and Qin Cheng ${ }^{1,2,3^{*}}$
}

\begin{abstract}
Background: Temotu Province, Solomon Islands is progressing toward malaria elimination. A baseline survey conducted in 2008 showed that most Plasmodium infections in the province were of low parasite density and asymptomatic infections. To better understand mechanisms underlying these malaria transmission characteristics genetic diversity and relationships among Plasmodium falciparum and Plasmodium vivax populations in the province were examined.

Methods: Forty-five P. falciparum and 67 P. vivax samples collected in the 2008 baseline survey were successfully genotyped using eight $P$. falciparum and seven $P$. vivax microsatellite markers. Genetic diversity, relationships and distribution of both $P$. falciparum and $P$. vivax populations were analysed.

Results: Plasmodium falciparum population exhibited low diversity with 19 haplotypes identified and had closely related clusters indicating clonal expansion. Interestingly, a dominant haplotype was significantly associated with fever and high parasite density. In contrast, the P. vivax population was highly diverse with 58 haplotypes identified that were not closely related. Parasite populations between different islands in the province showed low genetic differentiation.

Conclusion: The low diversity and clonal population of $P$. falciparum population may partially account for clinical immunity developed against illness. However, it is possible that importation of a new $P$. falciparum strain was the major cause of illness. High diversity in $P$. vivax population and low relatedness between strains suggested clinical immunity to $P$. vivax may be maintained by different mechanisms. The genetic diversity, population structure and distribution of strains indicate that transmission of $P$. falciparum was low, but that of $P$. vivax was still high in 2008. These data will be useful for assessing changes in malaria transmission resulting from interventions.
\end{abstract}

\section{Background}

Temotu Province is the most southern province in the Solomon Islands. The province has a population of approximately 22,000, residing on five main groups of islands: Santa Cruz (Ndendo), Reef Islands, Duff Islands, Utupua and Vanikoro. Malaria is endemic in Temotu Province with year-round transmission. Historically malaria is highly prevalent in Temotu province. Blood slide

\footnotetext{
* Correspondence: qin.cheng@defence.gov.au

'Drug Resistance and Diagnostics, Australian Army Malaria Institute, Weary

Dunlop Drive, Gallipoli Barracks, Enoggera, QLD 4051, Australia

${ }^{2}$ Pacific Malaria Initiative Support Centre, School of Population Health,

University of Queensland, Herston, QLD, Australia

Full list of author information is available at the end of the article
}

examinations (through active and passive case detections) conducted in eastern districts, including Makira and Santa Cruz, in 1971 revealed a slide positive rate of $36.7 \%$ [1]. Expansion of the malaria eradication programme during 1972-1974, with activities including cyclical spraying operations with DDT and mass drug administration, lowered the slide positive rate rapidly to $3.2 \%$ in 1974 [1]. Since then malaria incidence rate has remained relatively low in Temotu Province. In 2008, when the Solomon Island government raised the goal of its national malaria programme from control to elimination, Temotu Province was consequently identified as the first province targeted for malaria elimination. 
As a preliminary step towards elimination a baseline cross-sectional survey was conducted between October and November 2008 to obtain epidemiological information on malaria and vectors in the province. The blood survey covered approximately half of the population in the province and revealed an overall prevalence of Plasmodium spp. of $2.7 \%$ with Plasmodium vivax being the dominant species [2]. However, the parasite prevalence varied greatly between different islands with $11.6 \%$ on Duff Islands, $4.3 \%$ on Santa Cruz, and less than $2 \%$ on Utupua, Vanikoro and Reef Islands. The survey also revealed that 40 and $65.6 \%$ of microscopy-positive Plasmodium falciparum- and $P$. vivax-infected individuals respectively, had parasite densities below $100 / \mu \mathrm{L}$, and that $83.7 \%$ of P. falciparum and $97.1 \%$ of $P$. vivax-infected subjects were asymptomatic at the time of survey and remained asymptomatic during the week of survey [3]. PCR detected a significant subset of individuals carrying sub-microscopic parasitaemia, predicting an overall point prevalence of $8.7 \%$ for the province [3]. The findings of the survey provided a good epidemiological baseline of the malaria situation and spatial distribution of infections in the province. The survey results also raised several important questions: what is the underlying cause of asymptomatic malaria in this setting? Are parasite populations on different islands different?

Asymptomatic malaria, often associated with low parasite densities, is usually observed in adults living in high transmission areas after repeated infections of malaria [4-9]. It correlates with the development of exposurerelated clinical immunity to parasites. However, the high prevalence of asymptomatic malaria with low parasite density observed in Temotu Province occurred in areas of relatively low transmission intensity. Similar observations have been reported in other areas of low transmission, such as South America [10-15]. Asymptomatic malaria in these settings is difficult to explain by the relatively low number of exposures in this setting.

Clinical outcomes of malaria can be influenced by both parasite and host factors. Parasite factors include the pathogenesis of the parasite (density, cyto-adherence capability and pyrogenic threshold), the homogeneity of the parasite population and the relatedness of parasites within the population. While the pathogenesis properties of the parasite contribute directly to the clinical outcome of a patient, the homogeneity of parasite population and the relatedness of parasites influence the speed at which exposure-related and strain-specific immunity develops in the host population. It is possible that individuals living in low endemic areas develop clinical immunity after a small number of exposures and maintain the level of immunity due to low genetic diversity of the parasite population or close relatedness of the parasites. Host factors include erythrocyte polymorphisms, status of immune system and pregnancy.
In order to understand mechanisms underlying asymptomatic infections in Temotu Province, genetic diversity of both P. falciparum and P. vivax isolates collected during the 2008 baseline survey, their relatedness and distribution was analysed. The results shed light on the underlying cause of asymptomatic malaria in the province. From the malaria elimination aspect, asymptomatic malaria presents a major challenge because the large number of asymptomatic carriers can maintain malaria transmission and influence the transmission dynamics of malaria $[6,16,17]$, and are difficult to diagnose microscopically or by rapid diagnostic tests (RDTs). These results will also serve as baseline information for measuring and evaluating impact of interventions and progress made towards elimination.

\section{Methods}

\section{Study sites and sample collection}

\section{The 2008 baseline survey}

A mass blood survey was conducted in Temotu Province, Solomon Islands across the island groups of Santa Cruz (or Ndendo), Utupua, Vanikoro, Duff Islands and Reef Islands during October to November 2008 (Figure 1). The survey sites, consent process, sample collection methods, and malaria species detection and identification by microscopy are detailed elsewhere [2].

\section{DNA extraction and PCR speciation}

Extraction of genomic DNA from blood on filter papers and PCR to determine Plasmodium species were described previously [2]. DNA samples that were determined positive for $P$. falciparum and $P$. vivax were used for genotyping. Genomic DNA was also extracted from cultured P. falciparum lines of different origins and blood samples positive for $P$. vivax originating from other countries in the Pacific region, and used as positive and diversity controls for genotyping.

\section{Microsatellite genotyping of Plasmodium falciparum}

Eight microsatellite loci were genotyped using the primers described by $\mathrm{Su}$ and Wellems [18] and two round seminested PCR methods described by Anderson et al. [19] with some modifications to the PCR protocol including increasing cycle number by ten in each round. The microsatellite markers used were Polya, TA1, TAA60, TAA81, TAA87, TAA109, ARA2 and PfPK2. Their chromosomal locations and fluorescent labelling are detailed in Table 1.

For capillary electrophoresis, the second round labelled products were multiplexed in two combined dilutions: 1) Polyo, TA1, TAA60 and TAA81; and, 2) PfPK2, ARA2, TAA87 and TAA109, then run on an ABI 3100 Genetic Analyzer with POP7 at the QIMR Berghofer Medical Research Institute Scientific Services Analytic Facility. The length of PCR products was determined with reference to 


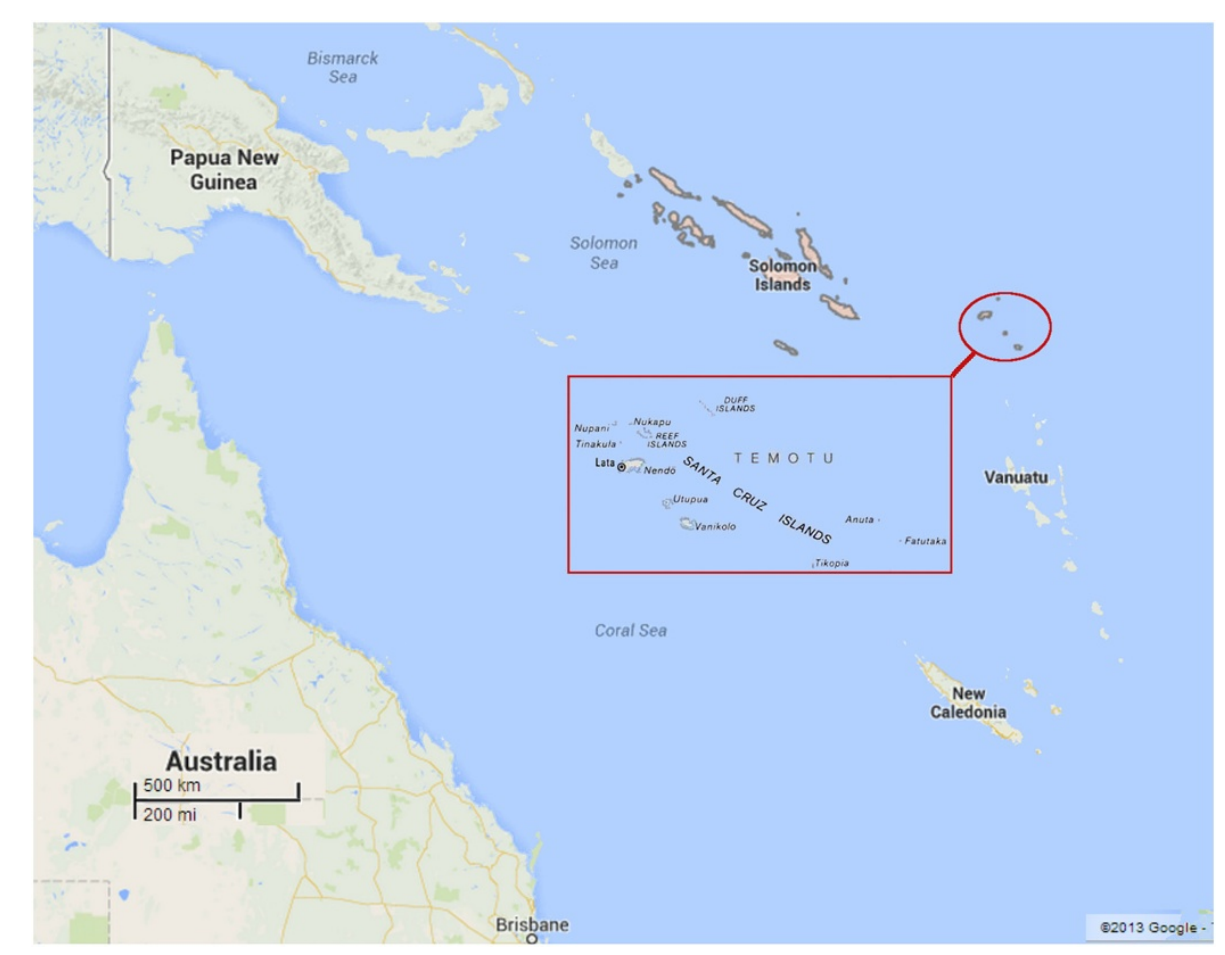

Figure 1 Map of Western Pacific region: geographical location of Solomon Islands and Temotu Province.

Table 1 Summary of microsatellite markers, number of alleles, cumulative number of haplotypes and expected heterozygosity $\left(H_{\mathrm{E}}\right)$ at each marker for Plasmodium falciparum and Plasmodium vivax isolates collected in Temotu Province

\begin{tabular}{|c|c|c|c|c|c|c|c|}
\hline & Locus & Chromosome location & Dye & $\mathbf{n}$ & No of alleles & $H_{\mathrm{E}}$ & $\begin{array}{c}\text { Cumulative number } \\
\text { of haplotypes }\end{array}$ \\
\hline \multirow[t]{9}{*}{$P f$} & Polya & 4 & PET & 45 & 7 & 0.52 & 7 \\
\hline & PfPK2 & 12 & NED & 43 & 7 & 0.52 & 16 \\
\hline & TAA87 & 6 & $\mathrm{VIC}$ & 45 & 5 & 0.59 & 17 \\
\hline & TAA81 & 5 & $\mathrm{VIC}$ & 45 & 5 & 0.71 & 19 \\
\hline & TA1 & 6 & 6FAM & 41 & 4 & 0.27 & 19 \\
\hline & TAA60 & 13 & NED & 44 & 4 & 0.55 & 19 \\
\hline & ARA2 & 11 & PET & 45 & 4 & 0.70 & 19 \\
\hline & TAA109 & 6 & $6 F A M$ & 44 & 3 & 0.46 & 19 \\
\hline & Mean \pm SE & & & 45 & $4.88 \pm 0.51$ & $0.54 \pm 0.05$ & \\
\hline \multirow[t]{8}{*}{ Pv } & MS8 & 12 & $\mathrm{VIC}$ & 60 & 20 & 0.94 & 20 \\
\hline & Pv3.27 & 3 & $6 F A M$ & 66 & 16 & 0.88 & 42 \\
\hline & MS16 & 9 & NED & 61 & 15 & 0.87 & 54 \\
\hline & MS10 & 13 & PET & 58 & 12 & 0.89 & 54 \\
\hline & MS5 & 6 & NED & 47 & 9 & 0.83 & 58 \\
\hline & msp1F3 & 7 & $\mathrm{VIC}$ & 62 & 7 & 0.79 & 58 \\
\hline & MS1 & 3 & $6 F A M$ & 48 & 5 & 0.75 & 58 \\
\hline & Mean \pm SE & & & 67 & $12 \pm 2.02$ & $0.85 \pm 0.02$ & \\
\hline
\end{tabular}


internal size standards ABI GS500 LIZ using Genescan and Peak Scanner software. Negative controls (no DNA and DNA from non-parasitised human blood) and standard strains (D6, W2 and TM96-C235) were included in each run for calibration between runs.

\section{Microsatellite genotyping of Plasmodium vivax}

Seven microsatellite markers were used to genotype $P$. vivax samples; this included three markers (Pv3.27, MS16 and msp 1F3) that required a two round nested PCR approach [20] and four (MS1, MS5, MS8 and MS10) that were single round PCR [21]. Their chromosomal locations and fluorescent labelling are detailed in Table 1.

The primary round amplification was performed as a multiplex of all three genotyping markers in a reaction volume of $25 \mu \mathrm{L}$ containing $3.2 \mathrm{mM} \mathrm{MgCl}, 0.24 \mathrm{mM}$ of each dNTP (0.96 mM total dNTPs), $0.2 \mu \mathrm{M}$ of each primer, $2.5 \mu \mathrm{L}$ of $10 \times$ ImmoBuffer and 0.625 units of Bioline Immolase $^{\mathrm{Tm}}$ DNA Polymerase with $1 \mathrm{uL}$ of template DNA added. The thermocycling profile was as follows: initial denaturation of $95^{\circ} \mathrm{C}$ for $10 \mathrm{~min}$, then 30 cycles of $95^{\circ} \mathrm{C}$ for $1 \mathrm{~min}, 56^{\circ} \mathrm{C}$ for $1 \mathrm{~min}, 72^{\circ} \mathrm{C}$ for $1 \mathrm{~min}$, and final extension of $72^{\circ} \mathrm{C}$ for $5 \mathrm{~min}$.

For the nested secondary round of amplification, each marker was amplified in a separate reaction in a volume of $25 \mu \mathrm{L}$ using fluorescence labelled reverse primers. The reaction mix contained $3.2 \mathrm{mM} \mathrm{MgCl} 2,0.16 \mathrm{mM}$ of each dNTP ( $0.64 \mathrm{mM}$ total dNTPs), $0.2 \mu \mathrm{M}$ of each primer, $2.5 \mu \mathrm{L}$ of $10 \times$ ImmoBuffer and 0.625 units of Immolase. One $\mu \mathrm{L}$ of the first round product was added as template. The thermocycling profile was identical to the first round.

The additional four microsatellite markers (MS1, MS5, MS8 and MS10) were typed using the methods described by Gunawardena et al. [21].

Both sets of labelled products were diluted and pooled before being run on an ABI 3100 Genetic Analyzer at the QIMR Berghofer Medical Research Institute Scientific Services Analytic Facility. The length of PCR products was determined with reference to internal size standards ABI GS500 LIZ using Genescan and Peak Scanner software. Negative controls (no DNA and DNA from nonparasitised human blood) and positive controls (PVQ, AMRU1 and AMRU2) were used in every run.

\section{Data analysis}

Alleles were scored manually using Peak Scanner Software ${ }^{\mathrm{Tw}}$ Version 1.0 (Applied Biosystems) using the height of 100 relative fluorescence units as the minimal peak threshold. The size range of the alleles and the average number of alleles per loci for each population were calculated.

\section{Multiplicity of infection (MOI)}

An infection was defined as polyclonal if there were two or more allele peaks present at one or more loci where the secondary peak was at least one-third the relative fluorescence unit height of the primary or highest peak [19]. MOI was calculated by dividing the total number of clones detected by the number of samples.

\section{Allele and haplotype frequency}

Allele frequency was calculated using only the predominant allele observed at each locus within each sample, while haplotype frequency was derived from combination of dominant allele type for each locus in each sample.

\section{Expected heterozygosity $\left(H_{E}\right)$}

As a measure of genetic diversity $H_{\mathrm{E}}$ was determined for each marker, based on the allele frequencies, defined by $H_{\mathrm{E}}=[\mathrm{n} /(\mathrm{n}-1)]\left[1-\Sigma \mathrm{p}_{\mathrm{i}}^{2}\right]$ where $\mathrm{n}$ is the number of samples and $\mathrm{p}_{\mathrm{i}}$ is the frequency of the $i$ th allele, using FSTAT (Version 2.9.3.2) [22]. For samples with polyclonal infections, only the predominant allele was used for this calculation. $H_{\mathrm{E}}$ values for both $P$. falciparum and $P$. vivax populations were also calculated across all markers for Temotu Province and within island groups. For this and subsequent statistical analysis the samples were divided into two island groups: 1) Santa Cruz, 2) outer islands including Reef, Duff Islands, Utupua and Vanikoro.

\section{Population differentiation $\left(F_{S T}\right)$}

Differentiation between populations, using pair-wise $F_{\mathrm{ST}}$, was calculated using FSTAT for comparing P. falciparum and $P$. vivax populations between the two island groups (Santa Cruz and outer islands including Reef, Duff Island, Utupua and Vanikoro) in Temotu Province.

\section{Spatial distribution of haplotypes}

Maps were generated using ESRI ArcMap9.2.

\section{Relationships between parasite haplotypes}

Phyloviz software [23] which implements the goeBURST algorithm [24], a refined version of the eBURST algorithm [25], was used to identify clusters of related haplotypes and generate a minimum spanning tree. Haplotype relationships were assessed using single locus variant (seven of the eight loci identical for $P$. falciparum and six of the seven loci identical for $P$. vivax) or double locus variant (six of the eight loci identical for $P$. falciparum and five of the seven loci identical for $P$. vivax).

\section{Results}

\section{Genetic diversity}

Overall, 8-loci-combined haplotypes were determined for 45 of $P$. falciparum. The median age of these subjects was 6.5 years (range from 1 to 60) with a median parasite density of $480 / \mu \mathrm{L}$ (range from 0 to $94,000 / \mu \mathrm{L}$ ). The number of alleles per locus ranged from three to seven with two markers (Polya and PfPK2) having the highest number of 
alleles (Table 1). Allele frequencies for each locus are illustrated in Figure 2. Combination of allelic types at four loci (Polyo, PfPK2, TAA87 and TAA81) produced a total of 19 haplotypes (PfH1-PfH19) from 45 P. falciparum samples examined (Table 1 and Figure 3). The number of haplotypes did not increase when four additional markers were typed (Table 1). Two haplotypes ( $\mathrm{PfH} 3$ and $\mathrm{PfH} 4$ ) consisted of 38 and $18 \%$ of the P. falciparum population while the remaining 17 haplotypes made up $2-7 \%$ each. The expected heterozygosity $\left(H_{\mathrm{E}}\right)$ for each locus ranged from 0.27 to 0.71 for $P$. falciparum with a mean of 0.54 $\left( \pm 0.05\right.$, Table 1). There was no significant difference in $H_{\mathrm{E}}$ of P. falciparum between Santa Cruz and outer islands (Mann Whitney test, $\mathrm{P}=0.55$ ).

For $P$. vivax, 7-loci-combined haplotypes were determined for 67 samples. The median age of these subjects was 6 years (range from 0 to 30) with a median parasite density of $240 / \mu \mathrm{L}$ (range from 0 to $12,230 / \mu \mathrm{L}$ ). The number of alleles per locus ranged from five for MS1 to 20 for MS8 (Table 1). MS8 also showed the most even distribution of different alleles with a highest allele frequency of $11.7 \%$ (Figure 2). Combination of allelic types at five loci (MS8, Pv3.27, MS16, MS10 and MS5) yielded a total of 58 haplotypes (PvH1-PvH58) in 67 samples examined. The number of haplotypes did not increase when additional two markers (MSP1F3 and MS1) were typed (Table 1). Of the 58 haplotypes, 51 (87.9\%) were seen once, each making up only $1.5 \%$ of the $P$. vivax population (Table 1 and Figure 3). The most frequent haplotype was seen three times constituting $4.47 \%$ of the population. The $H_{\mathrm{E}}$ value for each locus ranged from 0.75 to 0.94 for $P$. vivax with a mean of $0.85( \pm 0.02$, Table 1$)$. There is no significant difference in the $H_{\mathrm{E}}$ value for $P$. vivax between Santa Cruz and outer islands (Mann Whitney test, $\mathrm{P}=0.55$ ).

The overall $H_{\mathrm{E}}$ value of the $P$. vivax parasites was significantly higher than that of the sympatric $P$. falciparum parasites in Temotu Province (Mann Whitney test, $\mathrm{P}=0.0003$ ).
The same is true for both Santa Cruz and the outer island group when tested separately (Table 2).

\section{Multiplicity of infection in Plasmodium falciparum and Plasmodium vivax}

Two of the 45 P. falciparum isolates genotyped were two clone infections (detected on Polya, TA1 and PfPK2) giving a MOI of 1.04. In contrast, 20 of the 67 P. vivax samples examined had greater than one clone on any of the seven markers examined $(\mathrm{MOI}=1.33)$. The highest number of clones in one individual was three. While each of the seven markers detected at least one multiple clone infections, the combination of Pv3.27, msp1F3 and MS16 detected 80\% $(16 / 20)$ of the multiple clone infections in P. vivax.

\section{Shared haplotypes between island groups}

Two of the 19 P. falciparum haplotypes were observed on more than one island groups: $\mathrm{PfH} 3$, the most frequent haplotype, occurred on Santa Cruz $(\mathrm{n}=15)$ and Utupua/ Vanikoro $(n=2)$; PfH4 occurred on Santa Cruz $(n=6)$ and Duff /Reef Islands $(\mathrm{n}=2)$ (Figure $4 \mathrm{~A})$. In contrast, only two of the $58 P$. vivax haplotypes were shared between islands at the time of survey: $\mathrm{PvH} 27$ was observed once on Santa Cruz and twice on outer islands while PvH11 was observed once on Reef/Duff Islands and twice on Santa Cruz (Figure 4B).

\section{Differentiation of Plasmodium falciparum and Plasmodium} vivax populations between island groups

The $F_{\mathrm{ST}}$ values for the $P$. falciparum and $P$. vivax populations in the two island groups are shown in Table 3. $F_{\mathrm{ST}}$ value between the two island groups for $P$. falciparum and $P$. vivax populations was below 0.033 . The results indicate that both $P$. falciparum and $P$. vivax populations had low genetic differentiation between island groups. Therefore, there is no difference in parasite populations between Santa Cruz and outer islands in Temotu Province.

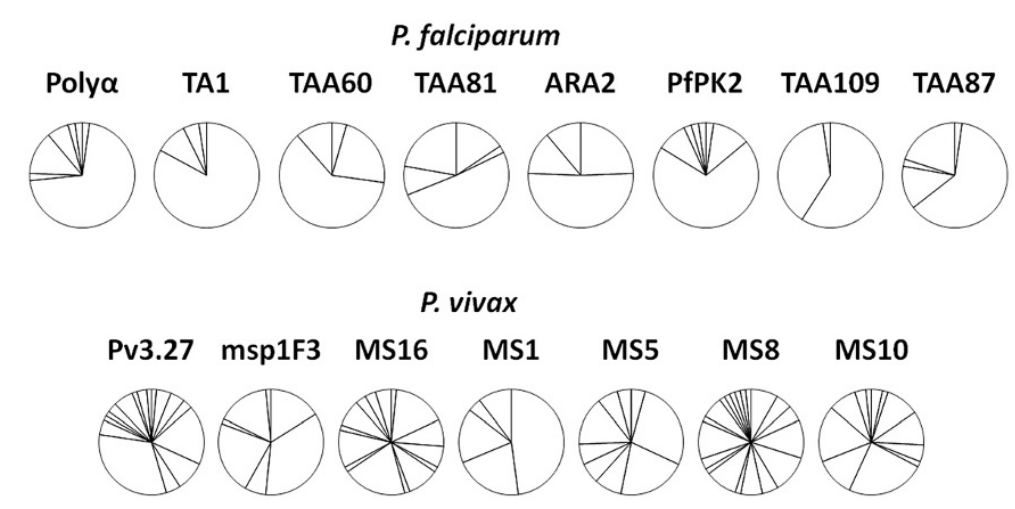

Figure 2 The number of alleles and allele frequency of the microsatellite markers examined for Plasmodium falciparum and Plasmodium vivax. 


\section{$\begin{array}{ll}\text { P. falciparum } & \text { P. vivax }\end{array}$}

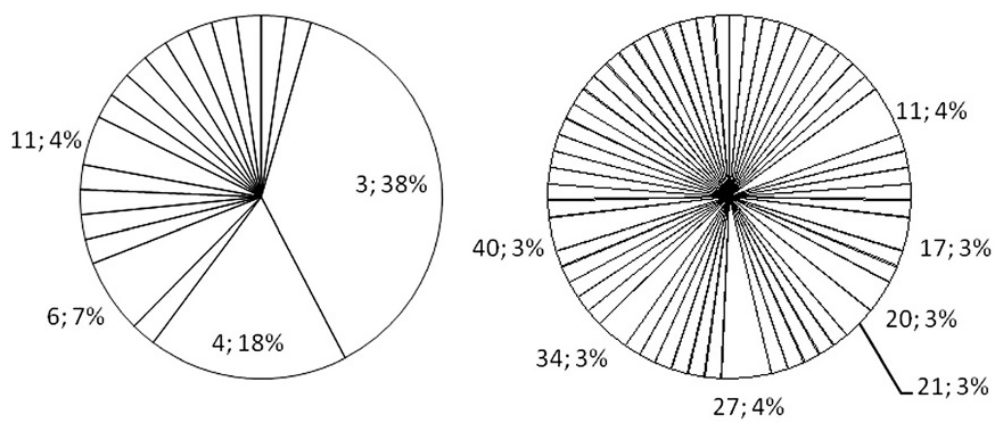

Figure 3 The number of haplotypes and haplotype frequency for Plasmodium falciparum and Plasmodium vivax. Only haplotypes with frequencies greater than $0.03, \mathrm{i}$ e, $>3 \%$, are indicated in the figure (haplotype; percentage).

Spatial distribution of Plasmodium falciparum and Plasmodium vivax haplotypes in Temotu Province Plasmodium falciparum appears to cluster in the northwestern side of Santa Cruz Island. Three dominant haplotypes PfH3, PfH6 and PfH11, were all identified in western coastal villages indicating a focal transmission (Figure 4A). PfH4 was also seen in two northern villages on Santa Cruz Island and the southern tip of Duff Island. In contrast, $P$. vivax was identified on both the north and south coast of Santa Cruz and on all other islands. Unique haplotypes, most identified only once, were scattered in different villages (Figure 4B).

\section{Genetic relatedness among haplotypes}

In $P$. falciparum haplotypes, four closely related clusters were identified: three clusters were formed by two single locus variants (identical on seven of the eight loci) and one cluster was formed by four double locus variants (identical on six of the eight loci). Each of the single locus variant clusters included one haplotype consisting more than $4 \%$ of the P. falciparum population indicating clonal expansion of these clusters. Two of these small clusters were observed on Santa Cruz, one shared between Santa Cruz and the Duff Islands, and one shared between Santa Cruz and the Reef Islands (Figure 5A).

For $P$. vivax, only one small cluster of single locus variant and five small clusters of double locus variants

Table 2 Comparison of $\boldsymbol{H}_{\mathrm{E}}$ between Santa Cruz and outer islands in Temotu Province

\begin{tabular}{|c|c|c|c|c|c|}
\hline \multirow[t]{3}{*}{ Island groups } & \multicolumn{2}{|c|}{ P. falciparum } & \multicolumn{2}{|r|}{ P. vivax } & \multirow{3}{*}{$\begin{array}{l}\text { p-value (Mann } \\
\text { Whitney test) }\end{array}$} \\
\hline & $n$ & $H_{\mathrm{E}}$ & $n$ & $H_{\mathrm{E}}$ & \\
\hline & & $($ Mean $\pm S E)$ & & (Mean \pm SE) & \\
\hline Santa Cruz & 36 & $0.53 \pm 0.04$ & 45 & $0.84 \pm 0.04$ & 0.0012 \\
\hline Outer islands & 9 & $0.56 \pm 0.06$ & 22 & $0.81 \pm 0.03$ & 0.0009 \\
\hline Total & 45 & $0.54 \pm 0.05$ & 67 & $0.85 \pm 0.02$ & 0.0003 \\
\hline
\end{tabular}

were identified. The single locus variant cluster contained two haplotypes that were each seen only once on the Duff Islands (Figure 5B). The five double locus variants, each contain two to three haplotypes, were mostly seen in Santa Cruz (Figure 5B).

\section{Relationship between haplotype and clinical and parasitological features}

Of the 45 P. falciparum samples analysed, eight were collected from febrile patients. From these symptomatic patient samples haplotype $\mathrm{PfH} 3$ was predominant (in six patients) and was significantly associated with fever (Fisher's exact test, $\mathrm{P}=0.0388$ ). It was followed by $\mathrm{PfH} 4$ and $\mathrm{PfH} 15$, in one patient each. PfH3 was also associated with high parasite densities as $29 \%(5 / 17)$ patients with $\mathrm{PfH} 3 \mathrm{had}$ parasite densities $\geq 10,000 / \mu \mathrm{L}$, compared to $3.6 \%(1 / 28)$ in non-PfH3 haplotypes (Fisher's exact test, $\mathrm{P}=0.028$ ). Age of individuals infected with $\mathrm{PfH} 3$ parasite (known for $13 / 17$ individuals) ranged from five to 56, with a mean of $24.54( \pm 4.21)$.

\section{Discussion}

Temotu Province, Solomon Islands is progressing towards malaria elimination. To better understand malaria epidemiology in this setting a baseline survey was conducted in 2008. The survey showed that most Plasmodium infections in the province were of low parasite density and asymptomatic infections [3]. The current study aims to examine genetic diversity and relationships among $P$. falciparum and $P$. vivax populations in Temotu Province in order to shed light on the mechanism underlying the observed malaria transmission characteristics in this setting.

A total of $45 P$. falciparum and $67 P$. vivax samples collected in the 2008 baseline study were analysed in this study. Like other epidemiology studies there is some unavoidable bias against samples with very small volume or very low parasite densities because of difficulties in 


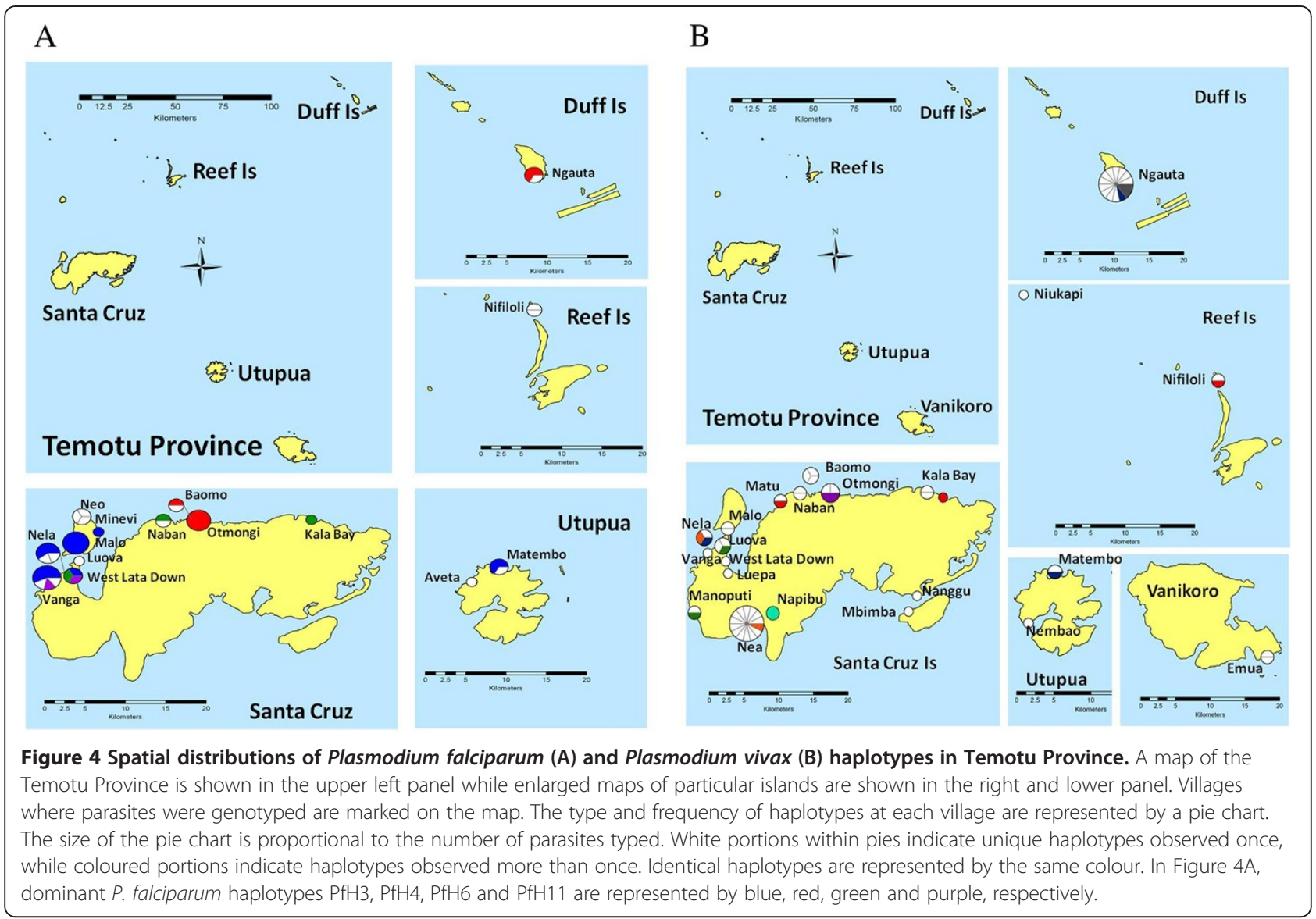

amplifying markers at multiple loci. Parasite density and subject's age appeared to be two major determinants for the success of genotyping on 7 or 8 markers. The median parasite density in samples successfully genotyped were 480 and 240 parasites/ $\mu \mathrm{L}$ compared to 10 and 16 parasites/ $\mu \mathrm{L}$, for $P$. falciparum and $P$. vivax, respectively, in those could not be genotyped (Mann Whitney test, $\mathrm{P}<0.0001$ ). The median age of subjects successfully genotyped were significantly younger than subjects not genotyped for both P. falciparum (6.5 vs 14, Mann Whitney test, $\mathrm{P}=0.02)$ and $P$. vivax (6 vs 9, Mann Whitney test, $\mathrm{P}=0.002$ ).

Genetic diversity among the $P$. falciparum and $P$. vivax populations in Temotu Province was compared by measuring diversity on eight and seven microsatellite markers, respectively. Most of these markers are neutral, located on different chromosomes, and have been widely used

Table $3 F_{\mathrm{ST}}$ values top half of table (bold) and p-values after 1000 permutations bottom half of table

\begin{tabular}{lccccc}
\hline Population & \multicolumn{2}{c}{ P. falciparum } & & \multicolumn{2}{c}{ P. vivax } \\
\cline { 2 - 3 } \cline { 5 - 6 } & Santa Cruz & Outer islands & & Santa Cruz & Outer islands \\
\hline Santa Cruz & - & $-\mathbf{0 . 0 1 6}$ & & - & $\mathbf{0 . 0 3 3}$ \\
Outer islands & 0.238 & - & & 0.126 & - \\
\hline
\end{tabular}

for population genetics studies [21,26,27]. For the $P$. falciparum population, results revealed 19 haplotypes in 45 samples analysed giving an average $H_{\mathrm{E}}$ of 0.54 , moderately diverse. The level of diversity for P. falciparum in Temotu Province is comparable to that reported in Thailand, higher than that in South America, but lower than that in Papua New Guinea (PNG) and African $P$. falciparum populations in 1990s [26] using similar but a larger set (12) of microsatellite markers.

In contrast, the $P$. vivax population was highly diverse. A total of 58 haplotypes were obtained in $67 P$. vivax samples, giving an average $H_{\mathrm{E}}$ of 0.85 , which was significantly higher than that in the sympatric $P$. falciparum population in the province, despite using one less marker (seven instead of eight). This high level of diversity is comparable to that reported in PNG [28], Tetere area, Solomon Islands [29], Kolkata [30], Sri Lanka and Myanmar [21], and higher than that reported for India, Thailand, Laos, Colombia [27] and Ethiopia [21] using similar markers. The findings are similar to that reported in Brazil where $H_{\mathrm{E}}$ for $P$. vivax and $P$. falciparum was determined to be 0.80 and 0.51 , respectively [31].

The P. falciparum parasites in the province showed some evidence of clonal expansion with four closely related 


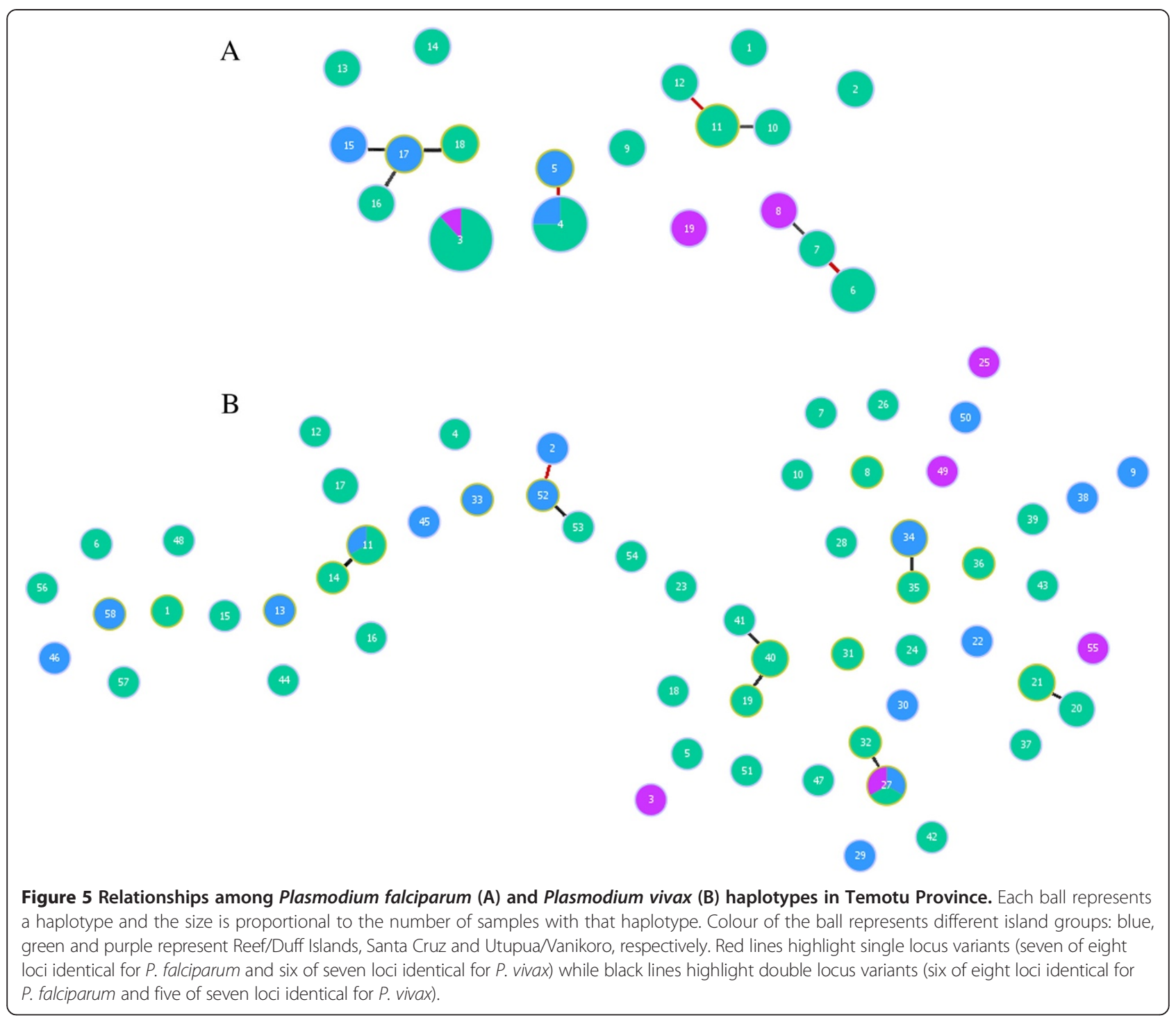

clusters representing $48.5 \%$ of $P$. falciparum population. These clusters, plus the most dominant haplotype PfH3 (38\%), represented $86.5 \%$ of the P. falciparum population in Temotu Province at the time of survey. Moreover, there was no evidence of a major bottle neck resulting in a loss of rare allele since $89 \%(17 / 19)$ of the haplotypes observed had a frequency below 0.1. In contrast, there was no evidence for clonal expansion in the $P$. vivax population. Only six small closely related clusters, involving 14 of the 58 haplotypes, were identified, representing only $33 \%$ of the $P$. vivax population.

Genetic diversity in parasite population is closely associated with transmission intensity of an area. High levels of genetic diversity are usually seen in areas with high levels of transmission where multiple clone infections are common, while as low diversity or clonal populations could be seen in areas with low transmission [32]. Genetic diversity levels observed in this study would suggest that the transmission intensity of $P$. falciparum in Temotu was low, but the transmission intensity of $P$. vivax was still high in 2008. This is in agreement with the prevalence data obtained during the survey where prevalence was 1.3 and $2.1 \%$ for $P$. falciparum and P. vivax, respectively [2]. This is also supported by higher MOI of $P$. vivax than $P$. falciparum (1.33 vs 1.04). While this difference in diversity between species could have resulted from differential selection in the history or differential susceptibility [33] of the only vector in the province, Anopheles farauti [34], it is also possible that the intervention measures were more effective in reducing transmission of $P$. falciparum than $P$. vivax, largely due to difficulties in curing the latent hypnozoite stage of $P$. vivax. The high diversity in $P$. vivax population is likely maintained by a large number of people carrying hypnozoites in their liver. 
Although a high rate of asymptomatic malaria was associated with both $P$. falciparum (83.7\%) and $P$. vivax (97.1\%) infections [3], the underlying mechanism is likely to be different between the two species. The P. falciparum population in Temotu Province has relatively low genetic diversity and parasites appear to be genetically closely related. This could speed up the development of acquired immunity to the $P$. falciparum population and thereby partially explain the high proportion of asymptomatic $P$. falciparum infections. In contrast, the $P$. vivax population was found to be highly diverse and not closely related. The large proportion of asymptomatic vivax malaria observed in the province could not be explained by lack of genetic diversity or the relatedness of the parasite population. This phenomenon has been increasingly reported in several areas with low transmissions [12-15]. It is possible that clinical immunity can be maintained with limited exposure and/or with prolonged infection at low parasite density; alternatively, other host factors may play a role in this phenomenon.

This study also provides important information for the malaria elimination programme in Temotu Province. Firstly, there was no significant differentiation of the parasite populations between Santa Cruz and the outer islands, and parasite haplotypes seen more than once were frequently found shared between different islands. These results suggest that there was relatively free movement of people carrying parasites among these islands. The provincial malaria elimination interventions would be more effective if coverage is for all islands.

Secondly, the study provides evidence for possible importation of $P$. falciparum strains. The $\mathrm{PfH} 3$ haplotype was the most dominant strain in the province consisting of $38 \%$ of the P. falciparum population. This strain was observed mostly in the western villages of Santa Cruz indicating focal transmission. PfH3 was significantly associated with fever as well as with high parasite densities. The focal distribution and illness associated with this strain suggest that the local host population lacks immunity against this strain of $P$. falciparum. This is further supported by the wide age range of the hosts infected with this strain, instead of predominantly children. Combined, these data indicate that PfH3 may be a newly imported parasite. This finding suggests that to eliminate malaria, vigilance should be maintained to prevent importation of parasites from other malaria areas.

Finally, this study provides baseline genetic diversity data for both P. falciparum and P. vivax populations in Temotu Province. Comparing future survey findings against these baseline data will help to assess any changes in parasite strains, genetic diversity and population structure. As these parameters are associated with malaria transmission intensity they will be useful for evaluating the impact of interventions and progress toward elimination.

\section{Conclusion}

The low diversity and clonal population of P. falciparum population may partially account for the high level of clinical immunity. However, it is possible that importation of a new $P$. falciparum strain has caused most of the symptomatic cases. In contrast, high diversity in the $P$. vivax population and low relatedness between strains suggested clinical immunity to $P$. vivax may be maintained by different mechanisms such as requiring limited exposures, prolonged infections with low parasite densities or host factors, rather than population homogeneity. The genetic diversity, population structure and distribution of strains indicate that transmission of $P$. falciparum was low, but that of $P$. vivax was still high in 2008. These data will be useful for assessing changes in malaria transmission resulting from interventions.

\section{Competing interests}

The authors declare that they have no competing interests.

\section{Authors' contributions}

$K G, S D$, and $L B$ performed experiment determining parasite genotypes; $K G$, $\mathrm{SD}, \mathrm{LB}$ and $\mathrm{QC}$ carried out data analysis; $\mathrm{AB}$ and LW contributed to the baseline survey; KG and QC wrote the manuscript; KG, DS and QC conceived and designed the study. All authors read and approved the final manuscript.

\section{Acknowledgements}

This project was partially funded by AusAID through Pacific Malaria Initiative Supporting Centre, University of Queensland. The authors would like to thank the survey participants of the Temotu Province, Solomon Islands. We are grateful to Dr Ingrid Felger for providing $P$. vivax microsatellite typing protocol, Wesley Sharrock for his assistance in optimizing $P$. vivax genotyping conditions and Dr Tim Anderson for advice on $P$. falciparum microsatellite markers. We thank Dr Michelle Gatton for providing statistical advice.

\section{Disclaimer}

The opinions expressed in this manuscript are those of the authors and are not to be interpreted as policy of the Australian Defence Force.

\section{Author details}

${ }^{1}$ Drug Resistance and Diagnostics, Australian Army Malaria Institute, Weary Dunlop Drive, Gallipoli Barracks, Enoggera, QLD 4051, Australia. ${ }^{2}$ Pacific Malaria Initiative Support Centre, School of Population Health, University of Queensland, Herston, QLD, Australia. ${ }^{3}$ Malaria Drug Resistance and Chemotherapy, QIMR Berghofer Medical Research Institute, Herston, QLD, Australia. ${ }^{4}$ Vector Borne Disease Control Programme, Ministry of Health, Honiara, Solomon Islands.

Received: 20 September 2013 Accepted: 7 November 2013 Published: 22 November 2013

\section{References}

1. Avery JG: Letter: The malaria eradication programme in the British Solomon Islands. P N G Med J 1975, 18:124-125.

2. The Pacific Malaria Initiative Survey Group: Malaria on isolated Melanesian islands prior to the initiation of malaria elimination activities. Malar J 2010, 9:218.

3. Harris I, Sharrock WW, Bain LM, Gray KA, Bobogare A, Boaz L, Lilley K, Krause D, Vallely A, Johnson ML, Gatton ML, Shanks GD, Cheng Q: A large proportion of asymptomatic Plasmodium infections with low and submicroscopic parasite densities in the low transmission setting of Temotu Province. Solomon Islands: challenges for malaria diagnostics in an elimination setting. Malar J 2010, 9:254.

4. De-Andrade AL, Martelli CM, Oliveira RM, Arias JR, Zicker F, Pang L: High prevalence of asymptomatic malaria in gold mining areas in Brazil [letter]. Clin Infect Dis 1995, 20:475. 
5. Imperato PJ: Malaria parasitemia in healthy Africans in North Mara, Tanzania. J Community Health 1986, 11:92-97.

6. Roper C, Elhassan IM, Hviid L, Giha H, Richardson W, Babiker H, Satti GM, Theander TG, Arnot DE: Detection of very low level Plasmodium falciparum infections using the nested polymerase chain reaction and a reassessment of the epidemiology of unstable malaria in Sudan. Am J Trop Med Hyg 1996, 54:325-331.

7. Steenkeste N, Rogers WO, Okell L, Jeanne I, Incardona S, Duval L, Chy S, Hewitt S, Chou M, Socheat D, Babin FX, Ariey F, Rogier C: Sub-microscopic malaria cases and mixed malaria infection in a remote area of high malaria endemicity in Rattanakiri province. Cambodia: implication for malaria elimination. Malar J 2010, 9:108.

8. Suarez-Mutis MC, Cuervo P, Leoratti FM, Moraes-Avila SL, Ferreira AW, Fernandes $\mathrm{O}$, Coura JR: Cross sectional study reveals a high percentage of asymptomatic Plasmodium vivax infection in the Amazon Rio Negro area, Brazil. Rev Inst Med Trop Sao Paulo 2007, 49:159-164.

9. Dal-Bianco MP, Koster KB, Kombila UD, Kun JF, Grobusch MP, Ngoma GM, Matsiegui PB, Supan C, Salazar CL, Missinou MA, Issifou S, Lell B, Kremsner P: High prevalence of asymptomatic Plasmodium falciparum infection in Gabonese adults. Am J Trop Med Hyg 2007, 77:939-942.

10. Branch O, Casapia WM, Gamboa DV, Hernandez JN, Alava FF, Roncal N, Alvarez E, Perez EJ, Gotuzzo E: Clustered local transmission and asymptomatic Plasmodium falciparum and Plasmodium vivax malaria infections in a recently emerged, hypoendemic Peruvian Amazon community. Malar J 2005, 4:27

11. Alves J, Roque AL, Cravo P, Valdez T, Jelinek T, Rosario VE, Arez AP: Epidemiological characterization of Plasmodium falciparum in the Republic of Cabo Verde: implications for potential large-scale reemergence of malaria. Malar J 2006, 5:32.

12. Roshanravan B, Kari E, Gilman RH, Cabrera L, Lee E, Metcalfe J, Calderon M, Lescano AG, Montenegro SH, Calampa C, Vinetz JM: Endemic malaria in the Peruvian Amazon region of Iquitos. Am J Trop Med Hyg 2003, 69:45-52.

13. Cerutti C Jr, Boulos M, Coutinho AF, Hatab Mdo C, Falqueto A, Rezende HR, Duarte AM, Collins W, Malafronte RS: Epidemiologic aspects of the malaria transmission cycle in an area of very low incidence in Brazil. Malar J 2007, 6:33

14. Cucunuba ZM, Guerra AP, Rahirant SJ, Rivera JA, Cortes LJ, Nicholls RS: Asymptomatic Plasmodium spp. infection in Tierralta, Colombia. Mem Inst Oswaldo Cruz 2008, 103:668-673.

15. Alves FP, Durlacher RR, Menezes MJ, Krieger H, Silva LH, Camargo EP: High prevalence of asymptomatic Plasmodium vivax and Plasmodium falciparum infections in native Amazonian populations. Am J Trop Med Hyg 2002, 66:641-648.

16. Coleman RE, Kumpitak C, Ponlawat A, Maneechai N, Phunkitchar V, Rachapaew N, Zollner G, Sattabongkot J: Infectivity of asymptomatic Plasmodium-infected human populations to Anopheles dirus mosquitoes in western Thailand. J Med Entomol 2004, 41:201-208.

17. Schneider P, Bousema JT, Gouagna LC, Otieno S, van de Vegte-Bolmer M, Omar SA, Sauerwein RW: Submicroscopic Plasmodium falciparum gametocyte densities frequently result in mosquito infection. Am J Trop Med Hyg 2007, 76:470-474.

18. Su X, Wellems TE: Toward a high-resolution Plasmodium falciparum linkage map: polymorphic markers from hundreds of simple sequence repeats. Genomics 1996, 33:430-444.

19. Anderson TJ, Su XZ, Bockarie M, Lagog M, Day KP: Twelve microsatellite markers for characterization of Plasmodium falciparum from finger-prick blood samples. Parasitology 1999, 119:113-125.

20. Koepfli C, Mueller I, Marfurt J, Goroti M, Sie A, Oa O, Genton B, Beck HP, Felger I: Evaluation of Plasmodium vivax genotyping markers for molecular monitoring in clinical trials. J Infect Dis 2009, 199:1074-1080.

21. Gunawardena S, Karunaweera ND, Ferreira MU, Phone-Kyaw M, Pollack RJ, Alifrangis M, Rajakaruna RS, Konradsen F, Amerasinghe PH, Schousboe ML, Galappaththy GN, Abeyasinghe RR, Hartl DL, Wirth DF: Geographic structure of Plasmodium vivax: microsatellite analysis of parasite populations from Sri Lanka, Myanmar, and Ethiopia. Am J Trop Med Hyg 2010, 82:235-242.

22. Goudet J: FSTAT, a program to estimate and test gene diversities and fixation indices (version 2.9.3.2). http://www2.unil.ch/popgen/softwares/fstat.htm. 2001.

23. Francisco AP, Vaz C, Monteiro PT, Melo-Cristino J, Ramirez M, Carrico JA: PHYLOViZ: Phylogenetic inference and data visualization for sequence based typing methods. BMC Bioinformatics 2012, 8:87.
24. Francisco AP, Bugalho M, Ramirez M, Carrico JA: Global optimal eBURST analysis of multilocus typing data using a graphic matroid approach. BMC Bioinformatics 2009, 10:152.

25. Feil EJ, Li BC, Aanensen DM, Hanage WP, Spratt BG: eBURST: inferring patterns of evolutionary descent among clusters of related bacterial genotypes from multilocus sequence typing data. J Bacterio/ 2004, 186:1518-1530.

26. Anderson TJ, Haubold B, Williams JT, Estrada-Franco JG, Richardson L, Mollinedo R, Bockarie M, Mokili J, Mharakurwa S, French N, Whitworth J, Velez ID, Brockman $\mathrm{AH}$, Nosten F, Ferreira MU, Day KP: Microsatellite markers reveal a spectrum of population structures in the malaria parasite Plasmodium falciparum. Mol Biol Evol 2000, 17:1467-1482.

27. Imwong M, Nair S, Pukrittayakamee S, Sudimack D, Williams JT, Mayxay M, Newton PN, Kim JR, Nandy A, Osorio L, Carlton JM, White NJ, Day NP, Anderson TJ: Contrasting genetic structure in Plasmodium vivax populations from Asia and South America. Int J Parasitol 2007, 37:1013-1022

28. Koepfli C, Ross A, Kiniboro B, Smith TA, Zimmerman PA, Siba P, Mueller I, Felger I: Multiplicity and diversity of Plasmodium vivax infections in a highly endemic region in Papua New Guinea. PLoS Negl Trop Dis 2011, 5:e1424.

29. Koepfli C, Timinao L, Antao T, Barry AE, Siba P, Mueller I, Felger I: A Large Reservoir and Little Population Structure in the South Pacific. PLOS One 2013, 8:e66041.

30. Kim JR, Nandy A, Maji AK, Addy M, Dondorp AM, Day NP, Pukrittayakamee S, White NJ, Imwong M: Genotyping of Plasmodium vivax reveals both short and long latency relapse patterns in Kolkata. PLoS One 2012, 7:e39645.

31. Ferreira MU, Karunaweera ND, da Silva-Nunes M, da Silva NS, Wirth DF, Hartl DL: Population structure and transmission dynamics of Plasmodium vivax in rural Amazonia. J Infect Dis 2007, 195:1218-1226.

32. Branch OH, Sutton PL, Barnes C, Castro JC, Hussin J, Awadalla P, Hijar G: Plasmodium falciparum genetic diversity maintained and amplified over 5 years of a low transmission endemic in the Peruvian Amazon. Mol Biol Evol 2011, 28:1973-1986.

33. Collins WE, Sullivan JS, Nace D, Williams T, Sullivan JJ, Galland GG, Grady KK, Bounngaseng A: Experimental infection of Anopheles farauti with different species of Plasmodium. J Parasitol 2002, 88:295-298.

34. Bugoro H, Cooper RD, Butafa C, Iro'ofa C, Mackenzie DO, Chen CC, Russell TL: Bionomics of the malaria vector Anopheles farauti in Temotu Province. Solomon Islands: issues for malaria elimination. Malar J 2011, 10:133.

\section{doi:10.1186/1475-2875-12-429}

Cite this article as: Gray et al:: Population genetics of Plasmodium falciparum and Plasmodium vivax and asymptomatic malaria in Temotu Province, Solomon Islands. Malaria Journal 2013 12:429.

\section{Submit your next manuscript to BioMed Central and take full advantage of:}

- Convenient online submission

- Thorough peer review

- No space constraints or color figure charges

- Immediate publication on acceptance

- Inclusion in PubMed, CAS, Scopus and Google Scholar

- Research which is freely available for redistribution 